\title{
MedienPädagogik
}

www. medienpaed.com

Zeitschrift für

Theorie und Praxis

der Medienbildung

ISSN 1424-3636

\section{Private Computer in der Schule: Zwischen schulischer Infrastruktur und Schulentwicklung}

Mandy Schiefner-Rohs, Richard Heinen, Michael Kerres

\begin{abstract}
Der vorliegende Artikel diskutiert die Implikationen eines schulischen IT-Konzepts, das darauf basiert, dass die Schülerinnen und Schüler explizit von der Schule aufgefordert werden, ihre privaten Geräte mit in den Unterricht zu bringen (Bring your own devices, BYOD). In einer Fallstudie wurden schulische Akteure einer Gymnasialklasse in Deutschland gefragt, wie sich die Nutzung privater Geräte von Schülerinnen und Schüler im Unterricht, zu der sich die Klasse gemeinsam entschieden hat, auswirkt. Untersucht werden sollte, ob sich dabei Unterschiede zu bisherigen Ansätzen einer 1:1-Ausstattung an Schulen zeigen, bei denen jede Schülerin und jeder Schüler ein Gerät (i. d. Regel Laptop) nutzt, das von der Schule organisiert und administriert wird. Als ein Ergebnis dieser Fallstudie kann festgehalten werden, dass von den Befragten keine inhaltlichen oder didaktischen Unterschiede zwischen der Nutzung privater Geräte und einer 1:1-Ausstattung mit schulisch organisierten Laptops wahrgenommen werden. Es zeigt sich vielmehr, dass organisatorische Prozesse bei der Integration digitaler Medien in Schulen erleichtert werden: Der Gerätesupport ist nicht mehr in dem bisherigen Umfang erforderlich, Ressourcen werden frei, um Fokus auf den didaktischen Einsatz digitaler Medien im Unterricht zu legen. Implikationen dieser Weiterentwicklung schulischer Infrastruktur werden vorgestellt und in Diskussionen um mediengestützte Schulentwicklung eingebettet.
\end{abstract}

\section{Ausgangslage}

Es wird in letzter Zeit immer wieder die Frage gestellt, ob sich die Kluft zwischen schulischer und privater Nutzung digitaler Medien nicht überwinden liesse, wenn privat verfügbare Endgeräte für unterrichtliche Zwecke genutzt werden könnten. Unter dem international gebräuchlichen Stichwort «Bring your own device» (BYOD) werden Schülerinnen und Schüler aufgefordert, ihre privaten Geräte mit in die Schule zu bringen und dort zu Lernzwecken zu nutzen. Dies könnte aber nicht nur eine pragmatische Lösung des technisch geprägten Ausstattungsdilemmas sein; es sind durchaus auch didaktische und pädagogische Potenziale einer 1:1-Ausstattung denkbar, die zu veränderten Unterrichtshandeln im Sinne von Heterogenität führen können. Gleichzeitig sind mit einem solchen Medien- 
konzept vielfältige Herausforderungen verbunden (Kerres, Heinen u. Stratmann 2012). Denn wenn alle Lehrkräfte und Lernenden ihre privaten Geräte nutzen, entsteht eine heterogene Gerätelandschaft. Es ist zu fragen, welche Vor- oder Nachteile dies für das Lehren und Lernen im Schulalltag hat. Hierbei können verschiedene Ebenen adressiert werden:

- Ebene der Infrastruktur: Einbindung der Geräte in die schulische Infrastruktur,

- Ebene der mobilen Geräte: Lauffähigkeit von Software auf unterschiedlichen Geräten,

- Ebene der Nutzung im Schulalltag: Umgang der Lehrkräfte mit Heterogenität von Schülerinnen und Schülern sowie Geräten, Umsetzung verschiedener LehrLernszenarien,

- Ebene der Akzeptanz durch Schülerinnen und Schüler: Identifikation mit dem persönlichen Gerät, sozialer Ausgleich u. a.m.

Um die Tragfähigkeit des Konzeptes zu überprüfen bzw. hemmende und unterstützende Faktoren benennen zu können, wird im Folgenden eine erste Fallstudie vorgestellt und diskutiert, die sich einiger dieser Fragen annimmt.

\section{Persönliche digitale Medien im Schulalltag: eine Fallstudie}

Zu fragen ist, welche Beobachtungen im Zusammenhang mit der Nutzung der privaten Ausstattung der Schülerinnen und Schüler im Unterrichtsalltag gemacht werden können. Da es bisher keine dieser Ansätze gibt, sind bis dato auch wenig empirische Antworten auf diese Frage zu finden. Aus diesem Grund wurde zu einer ersten empirischen Annäherung ein Fallstudienansatz gewählt. Dieser bietet sich in einem wenig explorierten Feld an, um nähere Erkenntnisse über dieses Feld zu sammeln (Fatke 2003; Muno 2009), im vorliegenden Fall mit der Frage, wie digitale persönliche Medien im alltäglichen Unterricht an einer deutschen Schule von Schülerinnen und Schülern, Lehrpersonen und der Schulleitung eingeschätzt werden. Eine erste Forschungsfrage lauteten daher:

- Welche Vor- und Nachteile werden im Rahmen eines solchen Einsatzes von heterogener Technologie im Unterrichtsalltag durch schulische Akteure berichtet?

Es ist vorstellbar, dass beispielsweise Lehrpersonen durch den Einsatz unterschiedlicher Technologien überfordert sind und es zu chaotischen Unterrichtskonzepten kommt. Ebenso ist vorstellbar, dass das digitale Gerät für Schülerinnen und Schüler als Statusobjekt angesehen wird und so im Rahmen des Unterrichts auch soziale Spaltungen auftreten. Ebenso ist daher zu fragen:

- Welche Implikationen hat das persönliche von den Eltern individuell angeschaffte Gerät auf den Unterricht im Gegensatz zu Laptops, deren Anschaffung und Administration die Schule zentral gesteuert hat? 
Betrachtet man Diskussionen um den Einsatz persönlicher Geräte, so werden zum Beispiel Fragen hinsichtlich der sozialen Unterschiede, die an Gerätebesitz sichtbar werden können, offenbar. Aber gleichzeitig werden Fragen hinsichtlich Organisation und Administration gestellt: Können durch einen solchen Ansatz technischadministrative Probleme gelöst werden, so dass sich Lehrpersonen beispielsweise wieder verstärkt dem eigentlichen Kerngeschäft von Schule, dem Unterricht widmen können? Oder geht durch die unterschiedlichen Geräte und Besitzfragen genau dieser Aspekt verloren, da beispielsweise mehr Zeit gebraucht wird, bis die Geräte einsatzbereit sind? Und schliesslich kann man fragen:

- Welche Kompetenzen werden aus Sicht von Lehrkräften und Schulleitung auf Seiten von Schülerinnen und Schülern, aber auch von Lehrenden benötigt?

Die Umsetzung einer 1:1-Ausstattung ermöglicht vielfältige didaktische Arrangements in Richtung Heterogenität im Klassenzimmer (Posch u. Altrichter 2009; Eisenmann u. Grimm 2011). Dies setzt voraus, dass Lehrpersonen kompetent sind, diese auch einzurichten. Ebenso stellt ein solcher Ansatz verstärkte Anforderungen hinsichtlich technischer Kenntnisse an Schülerinnen und Schüler.

\subsection{Fallbeschreibung}

Näher untersucht wurde ein Gymnasium in Nordrhein-Westfalen, das aufgrund der Umsetzung eines Konzepts des privaten Endgeräts im Klassenzimmer gezielt ausgewählt wurde. Die Schule hat seit 2001 in jedem 7. Jahrgang ein bis zwei LaptopKlassen eingerichtet. Die Geräte wurden dabei von der Schule beschafft und administriert und von den Eltern finanziert. Dieses Laptop-Konzept konnte aus mehreren Gründen nicht ausgeweitet werden: Der steigende administrative Aufwand konnte von der Schule nicht mehr geleistet werden. Weiterhin lag eine grosse Unzufriedenheit der Schülerinnen und Schüler vor, da Innovationszyklen schneller ablaufen als Neuanschaffungen erfolgen können. («Die Laptops sind dann doof und alt!») In Vorbereitung einer Anschaffung gab es immer wieder aus Schulsicht schwierige Einigungsprozesse mit Eltern über Gerätetypen. Ebenfalls fielen hohe Kosten an, da in den Klassen hochwertige Businessgeräte zum Einsatz kamen.

Die Schulleitung entschloss sich im Frühjahr 2011 zu einer Schülerinnen- und Schülerumfrage, um zu überprüfen, ob bei den Schülerinnen und Schülern des künftigen 7. Jahrgangs die Bereitschaft bestünde, eigene, private Hardware für und im Unterricht zu nutzen. Als Ergebnis dieser Studie zeigte sich, dass im gesamten Jahrgang 7 eine 1:3-Ausstattung in allen Klassen realisiert werden könnte (Heinen u. Kresse 2011). Die Schule entschloss sich dazu, diejenigen Schülerinnen und Schüler, die Interesse an der Nutzung ihrer privaten Hardware zeigten, in einer Klasse zusammenzufassen und den Einsatz privater Geräte in dieser Klasse zu im- 
plementieren. Allerdings wurden von der Schule einschränkende Regeln aufgestellt, welche technischen Voraussetzungen (z. B. Betriebssysteme, Bildschirmgrösse u. Ä.) die Geräte haben müssten, die die Schülerinnen und Schüler verbindlich mit in Unterricht bringen. Diese Regeln sollten eine Weiterarbeit mit den Unterrichtskonzepten der Notebook-Klassen ermöglichen. Mit Beginn des Schuljahres 2011/12 wurde so die erste Klasse gestartet, die ihre eigenen, privaten Geräte mit in den Unterricht nimmt und nutzen kann.

\subsection{Methodische Umsetzung der Fallstudie}

Methodisch wurden unter dem Aspekt der oben erwähnten Forschungsfragen während eines Schulbesuchs im Frühjahr 2012 (im 2. Jahr des BYOD-Einsatzes in der Schule) sowohl Interviews gewählt als auch in zufällig ausgewählten Unterrichtsstunden Beobachtungen durchgeführt. Ziel dieser methodisch triangulativ angelegten Studie war es, ein möglichst umfassendes Bild der Implikationen der Geräteintegration in den Schulalltag zu zeichnen und Sichten verschiedener Anspruchsgruppen auf das Thema zu eruieren.

Da Schulleitungen einen grossen Einfluss auf die Umsetzung schulischer Medienprojekte haben (z. B. Prasse u. Scholl 2001), wurde die Schulleitung dieser Schule mit einem leitfadengestütztem Interview hinsichtlich der Relevanz von Medienarbeit an der Schule und der Entwicklung des BYOD-Ansatzes, zu Schulentwicklung und Kommunikation mit digitalen Medien sowie der Implikationen der Einführung des BYOD-Ansatzes auf den Schulalltag befragt. Das Interview fand an der Schule statt und wurde schriftlich protokolliert. Darüber hinaus wurden sechs leitfadengestützte Interviews mit zufällig ausgewählten Lehrpersonen (Fächer Erdkunde, Latein, Mathematik, Chemie, Deutsch sowie Englisch; N=6) geführt. Der Leitfaden für die Lehrpersonen enthielt Fragen hinsichtlich des Einsatzes der Geräte im Unterricht (Häufigkeit, Art und Weise), der Beobachtungen im Zusammenhang mit der Einführung der Geräte auf den Unterricht oder allfälliger Rollenveränderungen, Fragen hinsichtlich Heterogenität der Geräte sowie Fragen zu technischen Aspekten, die sich durch die privaten, nicht von der Schule administrierten Geräte im Unterrichtsalltag zeigen. Zufällig ausgewählte Schülerinnen und Schüler $(N=6)$ wurden in einem Gruppeninterview befragt. Der Leitfaden für das Gruppeninterview enthielt Fragen nach der Bedeutung des privaten Gerätes für die Schülerinnen und Schüler, Fragen nach dem unterrichtlichem Einsatz, eine generelle Einschätzung eigener Laptops, Fragen zu technischen Schwierigkeiten sowie der ausserschulischen Benutzung der Geräte. Zusätzlich wurden in je einer zufällig ausgewählten Chemie- und einer Deutschstunde Unterrichtsbeobachtungen durchgeführt. Fokus wurde dabei auf die Kooperation und Kollaboration der Schülerinnen und Schüler untereinander während des Geräteeinsatzes, auf tech- 
nisch ausgelöste Interaktion (Fehlerbeheben usw.) sowie auf die Interaktion mit der Lehrperson gelegt.

Alle mit einem Leitfaden geführten Einzel- und Gruppeinterviews wurden transkribiert und mittels der Software MaxQDA induktiv und deduktiv kodiert (siehe Anhang) und im Anschluss analysiert. Aufgrund des explorativen Charakters der Fallstudie wurden vor allem induktiv Kategorien aus dem vorliegenden Material heraus ad hoc gebildet, wenn innerhalb der Daten Zusammenhänge und Textstellen entdeckt wurden, zu denen eigene Kategorien passen (vgl. Kelle u. Kluge 2010, S. 70), ebenso wurden deduktiv die aus den Forschungsfragen abgeleiteten inhaltlichen Strukturierungen des Leitfaden aufgenommen. Dabei wurde das Material ungefähr zur Hälfte durchgegangen und die bis dahin auftauchenden Textstellen kategorisiert. Nach der Hälfte wurde nochmals eine kritische Reflexion der so gewonnenen Codes vorgenommen und geschaut, inwieweit diese trennscharf sind, sie unter deduktiv gesetzte Kodes oder ineinander subsumierbar sind oder als eigenständiger Code bestehen bleiben können (vgl. Anhang). Die so entwickelten Codes wurden dann zur weiteren Analyse verwendet und zu Oberthemen zusammengefasst, die im nächsten Kapitel vorgestellt werden.

\section{Implikationen der Einführung persönlicher Geräte auf Unterrichtsentwicklung}

Die gefundenen Ergebnisse und Beobachtungen der Einführung persönlicher Geräte auf die Unterrichtsentwicklung werden im Folgenden aus der Perspektive unterschiedlichen Zielgruppen vorgestellt: So wird in einem ersten Schritt Lehrerhandeln mit persönlichen Geräten der Schülerinnen und Schüler betrachtet, um daran anschliessend die Perspektive der Schülerinnen und Schüler zu beleuchten. In einem dritten Schritt wird dann organisationales Handeln unter dem Konzept der Nutzung persönlicher Geräte betrachtet werden.

\subsection{Veränderungen auf das Lehrerhandeln im Unterricht}

Die Veränderungen durch Implementierung des BYOD-Ansatzes auf das Lehrerhandeln werden von Lehrpersonen vor allem mit dem Stichwort «Flexibilität« thematisiert: Sie müssen den Unterricht flexibler planen, da sie nicht von einheitlicher Software und Hardware ausgehen können. Durch die nicht von der Schule administrierten Geräte steigt aus Sicht der Lehrpersonen die Unsicherheit, welche Programme genau auf den Rechnern installiert sind. Während es in den bisherigen Laptopklassen einheitliche Geräte mit gleicher Konfiguration gab, ist dies bei den personalisierten Geräten anders. Damit steigt der Vorbereitungsaufwand für Lehrpersonen, da immer auch Alternativen mit vorbereitet werden müssen, bzw. der Verlauf von der Kreativität der Schülerinnen und Schüler in der Auswahl von Werkzeugen abhängt. Lehrende berichten vor allem von Schwierigkeiten, wenn Pro- 
gramme nicht laufen oder verfügbar sind (Chemielehrer, 13; Mathematiklehrerin, 14). Im Klassenzimmer gibt es jetzt nicht mehr nur Geräte eines Typs, die bei allen Schülerinnen und Schülern gleich konfiguriert sind, sondern mehrere unterschiedliche Formen und Ausstattungen. Somit entfällt nach Einschätzung der Lehrpersonen eine «spontane Problemlösung" oder auch der «Blick zum Nachbarn», wenn technische Probleme auftreten.

Interviewer: Haben sich Anforderungen an Ihre Kompetenzen, jetzt in dieser 1:1 der persönlichen Geräteausstattung verändert?

Lehrperson: Ja das geht so ein bisschen in diese Flexibilität hinein. Also sich mit unterschiedlichen Programmvarianten auseinander zu setzen. Aber das ist nichts, was nicht leistbar ist. Das geht relativ schnell. Ist ja häufig nur etwas andere Strukturen in den einzelnen Programmen. (Englischlehrer, 63-66)

Neben dieser Unsicherheit schätzen Lehrpersonen aber auch die didaktische Vielfalt, die ermöglicht wird, da sich daraus kreative Szenarien ergeben (Lateinlehrerin, 35-36) und Lehrpersonen sich immer wieder mit aktuellen Medienentwicklungen auseinandersetzen müssen (Chemielehrer, 65). Im Rahmen der Unterrichtsbeobachtungen wurden in zwei unterschiedlichen Unterrichtsstunden (Chemie und Deutsch) mediendidaktisch sinnvolle Settings beobachtbar, in denen die Geräte eine individuelle Auseinandersetzung mit dem Lernstoff förderten.

Die Abhängigkeit von Software ist fachbezogen unterschiedlich. Während die Mathematiklehrerin von veränderten Anforderungen spricht (Mathematiklehrerin, 18-19), sieht der Erdkundelehrer keinerlei Veränderungen auch hinsichtlich seiner Lehrerrolle (vgl. 47-48). Grund hierfür könnten unterschiedliche Anforderungen sein: Nutzung spezieller Mathematik-Software, die ggf. nicht für alle Gerätekonfigurationen verfügbar ist vs. Recherchen im Internet, die prinzipiell unabhängig von den technischen Ausstattungen der Geräte sind. Von einigen Lehrern wird die Technik in gewisser Weise als Unterrichtsgegenstand betrachtet (Chemielehrer, 84) oder bewusst auch technischer Inhalt (z. B. durch Informatiklehrer) vermittelt (Mathematiklehrerin, 97-100), andere Lehrer lehnen dies ab:

Ausser dass wir eben so kleine Probleme lösen, aber so in technische Details gehe ich als Erdkunde/ Politiklehrer nicht rein. (Erdkundelehrerin, 69-70).

Die neuen Herausforderungen, die sich an die Planung und Gestaltung von Unterricht ergeben, werden von Lehrerinnen und Lehrern unterschiedlich gross eingeschätzt. Während es Lehrpersonen gibt, die über eine höhere Medienkompetenz als andere Lehrpersonen zu verfügen scheinen und dementsprechend diesen Herausforderungen gelassen gegenüberstehen, wurde bei anderen Lehrpersonen vereinzelt der Wunsch geäussert, sich selbst im Bereich Medien weiter fortzubilden 
und in diesem Bereich mehr Kompetenzen zu erwerben (vgl. z. B. Deutschlehrerin, 27-28). Die meisten Lehrpersonen haben ein grobes technisches Verständnis, sowohl der Geräte als auch der Infrastruktur. Andere geben offen zu, «[...] weil ich mich begrenzt auch nur auskenne also ich kann jetzt nicht irgend so nen Programm herstellen nochmal, die technischen Kniffe fehlen teilweise, weil ich halt nicht kenne warum das jetzt hier nicht läuft [...]» (Englischlehrerin, 22).

Ansonsten gilt es in den Augen der Lehrpersonen, auch Verantwortung für Planung, aber auch Unterrichtsdurchführung und-ablauf abzugeben und sich mehr als Lernberater oder Moderator zu sehen (Englischlehrerin, 36-37). Diese Haltung und Veränderung der eigenen Rolle im Unterricht gilt aber nach Einschätzung mehrerer Lehrer sowohl für Laptop- als auch für BYOD Klassen, d. h. es ist keine Veränderung, die an die Besitzfrage und/oder die Unterschiedlichkeit des Gerätes gekoppelt ist und durchaus in anderen 1:1-Projekten beobachtbar ist (vgl. z. B. Häuptle 2006).

Fragt man Schülerinnen und Schüler nach der Kompetenz der Lehrpersonen im Umgang mit den digitalen Geräten, so nehmen auch diese unterschiedlich stark ausgeprägte Medienkompetenz hinsichtlich des Einsatzes auf Lehrerseite wahr. Dabei stimmen die Selbsteinschätzungen durch die Lehrpersonen hinsichtlich ihrer eigenen Kompetenz und die Fremdeinschätzung durch Schülerinnen und Schüler überein. Dies hat insofern Folgen für den Unterricht, als dass diese Lehrpersonen, die sich selber als weniger kompetent einschätzten, häufiger von Schülerinnen und Schülern vor allem hinsichtlich technischer Fragestellungen unterstützt werden.

\subsection{Veränderungen von Schülerinnen- und Schülerhandeln}

Die Veränderungen durch BYOD auf Schülerinnen- und Schülerseite können vor allem mit den Stichworten Verantwortung, Unterstützung sowie Vertrautheit zusammengefasst werden. So übernehmen Schülerinnen und Schüler nach Einschätzung der Lehrpersonen mehr Verantwortung für das eigene Gerät (Deutschlehrerin, Schulleitung, 15-16). Diese reicht aus Sicht der Lehrpersonen von der Verantwortung darüber, dass das Gerät im Unterricht einsatzfähig ist («aufgeladen und arbeitsbereit», Deutschlehrerin, 20) bis dahin, dass sie sich technisch besser auskennen, da es ihr persönliches Gerät ist und ihnen der Umgang durch den ausserschulischen Einsatz vertraut ist. In den Unterrichtsbeobachtungen fiel auch auf, dass die Arbeit mit den privaten Geräten den Schülerinnen und Schülern als sehr vertraut und selbstverständlich erschien. Die Geräte wurden wie Bücher herumgetragen oder auch zwischen Schülerinnen und Schülern weitergereicht. Ebenso übernehmen sie Verantwortung i.S., dass sie darauf achten, ihr Gerät nicht mit Viren zu infizieren oder anders zu beschädigen.

Darüber hinaus leisten Schülerinnen und Schüler gegenseitige Unterstützung, indem sie sich beispielsweise gegenseitig bei allfälliger Fehlersuche helfen, was so- 
wohl in den Unterrichtsbeobachtungen sichtbar war, aber auch von verschiedenen Lehrpersonen berichtet wurde (Erdkundelehrer, Lateinlehrerin, 37-38). Oft seien dies ja «[...] ganz triviale Dinge, die Kinder haben am Wochenende den Prozessor mal ausgeschaltet und kommen nicht mehr ins Netz oder sie haben ihr Passwort vergessen oder was auch immer. Häufig sind es ja Kleinigkeiten» (Erdkundelehrer, 29-30).

Wenn sich Störungen oder Probleme nicht mehr in angemessener Zeit beheben lassen, nutzen mehrere Personen einen einzigen Computer (vgl. Chemielehrer 20-21; Erdkundelehrer, 19), wobei dies nach Einschätzungen der Lehrpersonen nur eine Übergangslösung ist. Beispiele eines solchen Umgangs mit technischen Problemen konnten in der Unterrichtsbeobachtung mitverfolgt werden (UB 1, 9:10) Die Schülerinnen und Schüler schätzen es darüber hinaus, dass sie ihr Gerät selber pflegen können und auch damit spielen können. Dies birgt aber auch Ablenkungspotenzial für den Unterricht (Erdkundelehrer, 60; Englischlehrerin, 19). Dadurch, dass sich Schülerinnen und Schüler mit dem Gerät nun gut auskennen, gelingt es ihnen zum Teil einfacher, z. B. Spiele zu spielen, die mit zwei Tastaturgriffen vor dem Blick des Lehrers verschwinden (Mathematiklehrerin, 120-123). Vor allem am Anfang des Schuljahres haben Schülerinnen und Schüler das Potenzial eigener Medien genutzt und vermehrt über den im Klassenraum via Netzwerk zugänglichen Drucker gedruckt oder abseits des Unterrichtsthemas gesurft. Dieses Verhalten verschwand aber mit der Zeit. Selbst Schülerinnen und Schüler berichten von anfänglicher Euphorie, die sich allmählich legte. So haben sie beispielsweise Skype zum Chatten anfangs stark genutzt, was allerdings mit der Zeit seinen Reiz verlor (Schülerinnen und Schülerinterviews, 101-105). Allfällige Unaufmerksamkeiten werden durch die Lehrpersonen mit den Schülerinnen und Schülern thematisiert (Mathematiklehrerin, 125-126). Meist handelt es sich in Augen beider Gruppen um klare «Regelverstösse», deren Konsequenzen den Schülerinnen und Schülern klar sind (Schülerinnen und Schülerinterview, 54-57). So gibt es die Möglichkeit, das Internet auszuschalten, als "eine Strafe für die ganze Klasse», die allerdings noch nicht eingesetzt worden ist. Jedoch sind in Einzelfällen die Geräte einheitlich geschlossen worden - «dann einfach zuklappen lassen, wenn's dann nicht mehr ging» (Lateinlehrerin, 77-80).

Da die Schülerinnen und Schüler das Gerät bereits vor dem Start des Schuljahres besassen, fiel der Reiz des Neuen und die Ablenkung dadurch weg (Vertrautheit).

Aber am Anfang, das könnte auch noch der Vorteil von den individuellen Geräten sein, wenn die Eltern den Kindern die sag ich mal zu Beginn der Sommerferien schenken würden, haben sie etwa sechs Wochen um die Dinger auszuprobieren. Jetzt [Anmerkung der Autoren: in den alten Klassen ohne BYOD] wars so, dass sie natürlich in der Schule die Teile erst mal aus- 
probiert haben, alles war neu, alles war toll, dann musste im Matheunterricht natürlich ICQ runter geladen werden. Oder sowas. Also dass sie diese Dauer bis das Ding dieses Neuigkeitsinteresse verliert, das die nicht unbedingt auf die Schulzeit oder in die Schulzeit mitgebracht wird (Mathematiklehrerin, 49-50).

Der Einstieg in den Unterricht beginnt damit früher als in vergleichbaren LaptopProjekten, in denen Schülerinnen und Schüler den Laptop erst zu Beginn des Schuljahres erhalten haben. Schülerinnen und Schüler sind so nach Auskunft der Lehrpersonen stärker auf den Unterricht konzentriert und es vergeht weniger Zeit mit der Einführung in die Handhabung der Geräte. Diese wurde frühzeitiger erworben und der Neuigkeitseffekt wirkt sich nicht störend auf den Unterricht aus.

Statusunterschiede aufgrund unterschiedlicher Hardware-Ausstattungen und daraus ableitende Diskussionen bei Schülerinnen und Schülern konnten im Rahmen der vorliegenden Fallstudie nicht eruiert werden. Zwar gibt es Hinweise, dass Schülerinnen und Schüler unterschiedliche Ausstattungen wahrnehmen, doch liegen diese meistens auf Ebene der Accessoires (also beispielsweise, welche Laptoptasche oder welche Maus man hat, bzw. wie das Geräte individuell verziert wird. Das betrifft sowohl den Desktop mit Hintergrundbildern als auch den Gerätedeckel mit Aufklebern.) Damit zeigt sich eine Wahrnehmung vor allem im Bereich Personalisierung der Geräte (vgl. auch Ludwig, Mayrberger u. Weidmann 2011). Die Geräte an sich spielen eine eher untergeordnete Rolle. Dies kann zum einen damit erklärt werden, dass die Geräteunterschiede bei Schülerinnen und Schülern aufgrund mangelnder Kenntnis als nicht wichtig erachtet werden, zum anderen aber auch damit, dass es sich um einen Fall handelt, in dem sich Eltern für eine Teilnahme aktiv entscheiden konnten, und somit Geräteunterschiede als Ausprägung von Statusunterschieden nicht so stark ins Gewicht fielen. So könnte durch die freiwillige Teilnahme eine Verzerrung entstanden sein, dass sich nur Eltern gemeldet haben, die über ein Gerät im Haushalt verfügen, dass die Kinder dann nutzen können.

\subsection{Implikationen auf schulische Organisationsprozesse}

Für das organisationale Handeln der Schule fällt auf, dass sich der Administrationsaufwand für die Geräte auf Seiten der Schule entscheidend verringert. Ziel des Konzepts der privaten Geräte im Unterricht war es aus Sicht der Schulleitung vor allem, den Arbeits- und Organisationsaufwand auf Seiten der Schule zu minimieren (Schulleitung, 23). Die 1:1-Ausstattung schulisch angeschaffter Laptops wurde vor zehn Jahren durch die Eltern angeregt (Schulleitung, 76-77), während die Umstellung auf BYOD aus Initiative der Schule geschah, um den administrativen Aufwand zu schmälern. So müssen nicht mehr alle Laptops der Klasse via Images mit der gleichen Software bespielt werden. Das Herunterladen von jeweiligen 
Softwareaktualisierungen und die Administration von Netzzugängen sind deutlich geringer geworden. Neu für alle Beteiligte ist, dass der Support, der von der Schule geleistet wurde, jetzt in der Verantwortung der Schülerinnen und Schüler und ihrer Eltern liegt. Von daher, so war die Vermutung, könnte es auf Seiten der Eltern eine verstärkte Ablehnung geben. Die Rückmeldungen der Eltern sind dennoch laut Auskunft der Lehrpersonen zu grossen Teilen positiv. Eltern schätzen vor allem, dass sie nicht mehr "gezwungen» werden, den Computer zu kaufen, den die Schule als Standardgerät vorschreibt, sondern eine Wahl haben und die in der Familie vorhandenen Geräte nutzen können. Zwar wird durch die Lehrpersonen zum Teil mehr Engagement der Eltern auch in Bezug auf die Technik gewünscht (Mathematiklehrerin, 37-38), im Grossen und Ganzen scheinen, nach Einschätzung der Schulleitung die Eltern aber im Gegensatz zu den schulisch vorgeschlagenen Geräten deutlich zufriedener mit der Nutzung selbst ausgesuchter privater Geräte zu sein.

Die Vorerfahrung mit 1:1 war nach den Analysen der Schulleitungs- und Lehrerinterviews für die Schule eine wichtige Voraussetzung, um BYOD administrativ und didaktisch umsetzen zu können. Diese erste 1:1-Phase hat sehr zur allgemeinen Qualifikation der Lehrpersonen beigetragen, auch wenn es immer noch unterschiedliche Kompetenzen im Umgang mit digitalen Medien gibt. Durch die Historie als Laptopschule war das Unterrichten mit den privaten Geräten der Schülerinnen und Schüler und damit einhergehend mit einer heterogenen Ausstattungslandschaft für die beteiligten Lehrkräfte keine grosse Umstellung, denn die Lehrpersonen sind ein persönliches Arbeitsgerät auf Seiten der Schülerinnen und Schüler gewohnt. Für andere Schulen bedeutet dies, dass bei der Einführung von BYOD-Ansätze mit Sorgfalt vorgegangen werden sollte und umfangreiche Vorarbeiten hinsichtlich Administration und Kompetenzentwicklung aller Beteiligten sichergestellt werden sollten. Hier schliessen sich für die Zukunft weitere Untersuchungsfragen an.

\subsection{Zusammenfassung der Fallstudie}

Führt man sich die Eingangs formulierten Forschungsfragen vor Augen, so fokussierten diese vor allem auf Vor- und Nachteile eines heterogenen Einsatzes von Technologie im Unterrichtsalltag, Beobachtung von Veränderungen durch privater Geräte auf den Unterricht sowie Kompetenzanforderungen auf Seiten von Schülerinnen und Schülern sowie von Lehrpersonen. Nach Analyse der von uns erhobenen Daten kann hinsichtlich dieser Fragen resümiert werden, dass das Konzept des persönlichen Gerätes im Klassenraum in grossen Teilen keinen inhaltlich/didaktischen Unterschied zur bisherigen 1:1-Ausstattung mit schulisch organisierten Laptops aufweist. Private Geräte, die in den Unterricht integriert werden, erleichtern vor allem organisatorisch/strukturelle Prozesse für Schulen. Damit steht der 
Support von Laptops nicht mehr auf oberster Agenda, sondern man verwendet die meisten ,Ressourcen' auf Fragen des didaktisch orientierten Einsatzes der Geräte in den Schulunterricht. So galten bisher die Betreuung technischer Geräte sowie die dadurch hervorgerufenen zeitlichen Belastungen einzelner Lehrer oder das Nicht-Vorhandensein technischer Administratoren als bedeutende Hemmnisse der Integration digitaler Medien in die Schule (Weinreich u. Schulz-Zander 2000; Schaumburg 2002). Diese Hemmnisse können durch den Einsatz privater Geräte in grossen Teilen beseitigt werden. Schulische Administratoren sind nur noch für die Integration der Geräte in die schulische IT-Landschaft, nicht aber für deren Wartung und Support zuständig, was zu einer Entlastung auf Seiten der Schule und einzelner Lehrkräfte führt und den Blick für andere Aufgaben freimachen kann. Lehrpersonen sind durch heterogene Geräte im Schulalltag angehalten, den Unterricht so zu planen, dass flexible Einsatzmöglichkeiten gegeben sind. Im Unterricht selbst sind sie zum Teil mit nichtfunktionierender Technik konfrontiert, für die sie aber ausgehend von vorherigen Projekten Handlungsoptionen aufgebaut haben. Vergleicht man die Probleme, die im Rahmen des classroom managements aus Sicht der Lehrpersonen auftauchen mit denen bei der Einführung digitaler Medien in Schulen, so sieht man, dass diese ähnlich geblieben sind (vgl. z. B. Sandholtz, Ringstaff u. Dwyer 1997). Lehrpersonen im vorliegenden Fall können aufgrund ihrer Kompetenzen auf Stufe des mastery eingeordnet werden: Sie verfügen über Strategien, bei Problemen mit den privaten Laptops oder unangemessenem Schülerinnen- und Schülerverhalten angemessen zu reagieren.

Schülerinnen und Schüler wünschen sich eher eigene private Geräte statt schulisch vorgeschlagene. Mit ihren eigenen Geräten kennen sie sich gut aus und sind mit ihnen vertraut. Diese Vertrautheit spiegelt sich im Unterricht in einer «Alläglichkeit» wider, d.h. den Geräten wird oftmals weniger Stellenwert zugewiesen, so dass sie als ganz normales Arbeitsgerät in den Unterricht integriert werden. Die eigene oder mit den Eltern getroffene Entscheidung für ein bestimmtes Gerät und die finanzielle wie administrative Verantwortung spielen für die Schülerinnen und Schüler eine wichtige Rolle.

Zusammenfassend können folgende Aussagen für den vorliegenden Fall generiert werden: Es zeigen sich kaum didaktisch-inhaltliche Unterschiede in der Nutzung privater Geräte im Unterricht zu bisherigen Laptopklasssen. Unterschiede werden vor allem in folgenden Punkten gesehen:

- Verantwortung und Unterstützung: Schülerinnen und Schüler übernehmen mehr Verantwortung für das eigene Gerät und die Ausstattung ihrer Mitschülerinnen und Mitschüler. Sie schätzen es, das Gerät selbst pflegen zu können, es immer verfügbar zu haben und auch damit spielen zu dürfen. 
- Vertrautheit: Da die Schülerinnen und Schüler das Gerät bereits vorher besitzen, fallen der Reiz des Neuen im Unterricht weg. Der Einstieg in die reguläre unterrichtliche Nutzung beginnt früher als beim Einsatz von schulischen Geräten.

- Flexibilität: Lehrkräfte müssen den Unterricht flexibler planen, da sie nicht von einheitlicher Software ausgehen können', schätzen aber auch die Vielfalt, da sich daraus kreative Szenarien ergeben. Die Abhängigkeit von Software ist evtl. fachbezogen. Die neuen Herausforderungen werden von Lehrpersonen unterschiedlich gross eingeschätzt.

- Aufwand: Der Administrationsaufwand der Geräte durch die Schule verringert sich.

Einschränkend muss hinzugefügt werden: Die Vorerfahrung mit 1:1 war für die Schule eine wichtige Voraussetzung, um die privaten Endgeräte administrativ und didaktisch umsetzen zu können. Die 1:1-Phase hat sehr zur Qualifikation der Lehrkräfte beigetragen. Eine Skepsis hinsichtlich veränderter Anforderungen an Lehrpersonen können im Rahmen der zugrundeliegenden Fallstudie nicht unbedingt bestätigt werden. Zwar verändern sich die Kompetenzanforderungen an Lehrpersonen und werden von diesen auch als herausfordernd gesehen, dennoch berichten die Befragten von guten Umgangsstrategien mit diesen Herausforderungen. Dies mag an dem besonderen Kontext der Fallstudie liegen, wurde doch hier eine Schule beobachtet, die schon 10 Jahre Erfahrung mit einer 1:1-Ausstattung im Unterricht hat. So ist davon auszugehen, dass hier die von Fullan (2001) gezeigten Innovationszyklen von «initation, implementation und continuation, routinization, or institutionalization» bereits weitgehend durchlaufen wurden und die Ausweitung des 1:1-Ansatzes hinsichtlich der Diversität der Geräte kein grosser inhaltlicher Sprung ist, sondern einen konsequenten evolutionären Schritt darstellt.

Gleichzeitig muss auf die Limitierung der Ergebnisse durch das methodische Design hingewiesen werden. In der vorliegenden Fallstudie wurden in einem festgelegten Setting verschiedene Akteure einer Schule befragt, die BYOD als Konzept im Rahmen einer Jahrgangsstufe einsetzen. Von daher sind die vorliegenden Ergebnisse nicht auf andere Schulen und Fälle verallgemeinerbar. Die untersuchte Schule kann schon als «selbst erneuernde Medienschule» (Schnoor 1998) bezeichnet werden, die Wege gefunden hat, neue technische Innovationen in bestehende Konzepte zu integrieren. Dennoch zeigen diese ersten Ergebnisse, welche Impli-

1 Hinsichtlich der zu installierenden Software werden von der Schule folgende Angaben gemacht: «Eins der Microsoft-Windows-Betriebssystem Windows XP oder Windows 7 oder aber das AppleBetriebssystem Mac OS X muss installiert sein. Android, iOS oder Windows 8 sind nicht zulässig. Ein automatisch updatendes Antivirussystem ist unabdingbar. Die Microsoft-Office-Produkte Microsoft Word, Microsoft Excel und Microsoft PowerPoint in der Version 2007 oder 2010 bilden die Grundlage für den Aus- tausch selbst erstellter Dokumente und sind unverzichtbar. Alle anderen nötigen Programme sind kostenlos. Produktnamen und Bezugs- quellen werden bekannt gegeben.» (Schulinformationsblatt) - Nach den positiven Erfahrungen im ersten Jahr wurden diese engen Regelungen im Folgejahr aufge-weicht. 
kationen persönlich angeschaffte, mobile Geräte und die dadurch entstehende Heterogenität im Klassenzimmer auf den Unterricht und die Schule haben können. Ebenso werden Strategien sichtbar, wie Lehrpersonen mit einer heterogenen Geräteausstattung im Unterricht umgehen. Wichtiger werden für sie eine gute und sorgfältige Vorbereitung sowie kreativer Umgang mit möglichen technischen Hindernissen.

Es sind aber weitere empirische Untersuchungen erforderlich, um mehr über die Implikationen privater Endgeräte und der Bedeutung von Gerätevielfalt im Klassenzimmer, vor allem für die Unterrichts- und Schulentwicklung zu erfahren. Forschungsfragen wären beispielsweise: Wie verändert sich Lernen im Unterricht, wenn unterschiedliche Geräte in der Klasse vorhanden sind? Welche Implikationen hat eine "pervasive education» für Schülerinnen und Schüler, wenn über das BYOD-Konzept digitale Endgeräte in allen Klassen und nicht nur in ausgewählten Projektklassen verfügbar sind? Nichtsdestotrotz kann und soll an dieser Stelle ein Ausblick auf mögliche Implikationen auf Schule und Schulentwicklungen skizziert werden.

\section{Diskussion: Mögliche Implikationen der Einführung von BYOD auf die Schule}

Seit der ersten Erprobung von Computern in der Schule in den 1960er Jahren haben sich Einsatzfelder und Zielsetzungen der Computernutzung immer wieder verändert und erweitert (Schulz-Zander 2001). Ein wichtiger Schritt, um intensiver mit Computern im Klassenzimmer arbeiten zu können, wurde mit der Einführung so genannter Notebook- oder Laptopklassen etwa ab der Jahrtausendwende getan. Leitend bei der Implementierung dieser Notebook-Klassen waren zwei Erkenntnisse, die auch für die Weiterentwicklung i.S. der Integration persönlicher Endgeräte in den Unterricht zentral sind: Die Durchdringung aller Bereiche der Lebens- und Arbeitswelt mit digitalen Medien erfordert eine Vorbereitung in der Schule, die über Informatikunterricht und informationstechnische Grundbildung hinausgeht (BMBF 2010). Und ein sinnvoller, situativer Einsatz digitaler Medien ist nur möglich, wenn Technik jederzeit im Klassenzimmer verfügbar ist (Petko 2012). In diesen Notebook-Klassen verfügen die Lernenden über Laptops, die im Unterricht und Zuhause eingesetzt werden. Während z. B. in den USA grosse Initiativen komplette Jahrgänge in ganzen Bundesstaaten mit staatlich finanzierten Geräten ausstatteten², beschränkten sich Notebook-Klassen in Deutschland zunächst auf einzelne Klassen in ausgewählten Schulen (Vorndran u. Schnoor 2003). Einzelne Notebook-Projekte wurden durch zeitlich und finanziell beschränkte Landesiniti-

2 Im internationalen Vergleich ist festzustellen, dass Initiativen zur Ausstattung mit personalisierten Endgeräten überwiegend staatlich finanziert werden. Hierzu gehören neben grossen Initiativen etwa in den USA auch OLPC-Projekte in verschiedenen Schwellenländern (Nugroho u. Lonsdale 2010). 
ativen vorangetrieben und umgesetzt. In Deutschland gibt es zwar immer wieder politische Lippenbekenntnisse für eine 1:1-Ausstattung (Deutscher Bundestag 2011), entsprechende langfristige Ausstattungsinitiativen fehlen aber - und ihre Finanzierung ist auf Dauer politisch nicht gewollt oder kaum realisierbar. Dabei stellt sich nicht nur die Frage nach der Finanzierbarkeit der Geräte, es fehlt auch an Schulen das Personal zur Administration. Gerade diese Frage der personellen Unterstützung der schulischen Medienarbeit hat weitreichende Folgen für den Einsatz von Laptops im Unterricht (Häuptle u. Reinmann 2006; Lampe et al. 2010). An diese Entwicklungen können persönliche Geräte in der Schule anknüpfen. Denn im Gegensatz zu dieser punktuellen Ausstattung mit Geräten für den individuellen Einsatz in der Schule ist der Alltag von Jugendlichen in hohem Masse medial durchdrungen und sie verfügen über eine bessere Ausstattung mit mobilen digitalen Endgeräten als je zuvor. Die JIM-Studien 2010 und 2011 (MPFS 2010 u. 2011) zeigen, dass Jugendliche zu Hause fast ausnahmslos auf Computer und Internet zugreifen können. Auch die persönliche Ausstattung von Kindern und Jugendlichen stellt sich als sehr gut dar. Das im Artikel vorgestellte «Bring your own device» ist ein Konzept, das versucht, die privat gute Ausstattung von Kindern und Jugendlichen auch im schulischen Kontext zu nutzen. Während Befürworter Flexibilität der Ausstattung und eine leicht zu administrierende 1:1-Ausstattung hervorheben, gibt es auch vielfältige Kritik des Ansatzes: von der Gefahr der Manifestation sozialer Ungleichheit, der technologiegetriebenen Perspektive von didaktischen Entscheidungen bis hin zur Befürchtung einer Überforderung der Lehrpersonen. Zusammenfassend sind diese Befürchtungen in den Ergebnissen der vorliegende Fallstudie nicht sichtbar: Die Nutzung privater Geräte hat aus Sicht der befragten Schülerinnen und Schüler sowie Lehrpersonen weniger Auswirkung als zu Beginn befürchtet. Der Geräteeinsatz im Unterricht wird von allen Beteiligten als «alltäglicher» eingeschätzt, so dass die Besonderheit technischer Ausstattung nicht mehr im Vordergrund steht, sondern dass mobile Geräte ein Arbeitswerkzeug unter vielen wird, auf das situationsgebunden und spontan zugegriffen werden kann. Die Lehrpersonen fühlen sich wenig verunsichert und durch den Einsatz unterschiedlicher Geräte nicht zusätzlich belastet. Ebenso ist nicht feststellbar, dass sich die Nutzung der Geräte im Unterricht am «schlechtesten» Gerät im Klassenzimmer ausrichtet, wie es in meist Lehrerdiskussionen befürchtet wird. Bei technischen Schwierigkeiten stehen Schülerinnen und Schüler füreinander ein und helfen sich gegenseitig sowie den Lehrpersonen. Auch das Thematisieren von sozialen Ungleichheiten durch die Geräteausstattung wurde im vorliegenden Projekt nicht sichtbar.

Innerhalb der hier vorgestellten Fallstudie ist die Tendenz zu beobachten, dass digitale Geräte im alltäglichen schulischen Handeln eher in den Hintergrund treten.

Diese Veränderung trat aber nicht ad hoc ein, sondern benötigte verschiedene 
Entwicklungen: von einer 1:1-Ausstattung mit durch die Schule gestellten Geräten über eine Nutzung der persönlichen Geräteausstattung mit Mindestvorgaben verändert sich der Umgang mit den Geräten. Nach Pilotierung des BYOD-Konzeptes an der Schule ist die Tendenz zu beobachten, dass nach und nach auch die Mindestanforderungen und Restriktionen an die Geräte wegfallen. So ist im nächsten Schuljahr beispielsweise neben Windows auch Mac-Software erlaubt (während Tablets immer noch verboten sind). Hier ist eine langsame Entwicklung bzw. Anpassung im Rahmen der Medienausstattung zu beobachten: allmählich passen sich die Regeln für die Ausstattung der technischen Wirklichkeit an.

Doch was bedeutet die Integration privater Geräte in den Schulalltag, wenn man von dieser Fallstudie ausgehend weiterdenkt? Der Ansatz des Nutzens persönlicher Geräte im Unterricht bringt Veränderungen hinsichtlich der Verantwortlichkeiten und Ansätze von Psychological Ownership (Pierce, Kostova u. Dirks 2001) mit sich, die weiter untersucht werden sollten. Digitale Medien in der Schule sollten eigentlich angesichts der Medienentwicklung in der Gesellschaft alltäglich sein. Dennoch verwundert es, dass digitale Medien sich immer noch nicht so recht an Schulen integriert haben und zum selbstverständlichen Teil des Methodenrepertoires im Unterricht geworden sind. Aus der Innovationsforschung weiss man, dass unter anderem der wahrgenommene Nutzen die Komplexität bzw. Einfachheit der Nutzung, die Sichtbarkeit für anderen und Kompatibilität als zentrale Momente gelten, dass sich Innovationen in Organisationen durchsetzen (Rogers 2005, S. 265ff.). Hier kann das Konzept des «Bring your own device» ansetzen, denn es ist sowohl für Schülerinnen und Schüler als auch für Lehrpersonen «einfach», die eigenen Geräte im Unterricht zu nutzen. Diese drei Aspekte werden bei einer Nutzung des persönlichen, d. h. des vertrauten Gerätes wichtig. Ging man bei der Integration digitaler Medien zu Beginn davon aus, dass die Schule oder der Bildungsträger diese Geräte zu stellen hat, damit viele monetäre und personale Investitionen auch in den Aufbau dieser Infrastruktur geflossen sind, werden mehr und mehr die Individuen in den Fokus genommen. Die Ausstattung der Schülerinnen und Schüler mit digitalen Geräten wird damit in die Hände der Eltern gelegt und die persönlichen Geräte, seien es Laptops, Netbooks oder Tablets, werden zu Arbeitsinstrumenten ähnlich Heft und Stifte ${ }^{3}$.

Unter einer grösseren Perspektive betrachtet kann die Mediennutzung durch die Integration privater Geräte in den Schulalltag Implikationen auf allen Ebenen der Schulentwicklung haben (Rolff 2007). Das Potenzial des Konzepts liegt weniger in der Diskussion um Ausstattung oder schulischer IT-Infrastruktur, sondern ist vielmehr in der systematischen Weiterentwicklung von Schule zu sehen, wenn es zielgerichtet und bewusst umgesetzt wird. Unter dieser grösseren Perspektive kann es

3 Für diesen sich abzeichnenden Trend sind verschiedene Entwicklungen ursächlich: Geringere Gerätepreise, deutliche Durchdringung des Alltags mit digitalen Medien und daher auch meist eine gute private Ausstattung. 
Einfluss auf alle drei Teilbereichen von Schulentwicklung (Unterricht-, Personal- und Organisationsentwicklung) haben: Durch die Integration unterschiedlicher Geräte sind Lehrpersonen angehalten, sich immer wieder mit tragfähigen Konzepten der Medienintegration in den Unterricht auseinanderzusetzen und neue technische Entwicklungen in den Unterricht zu integrieren. Sie müssen den Unterricht flexibler planen bzw. den Problemlösungskompetenzen der Schüler mehr Raum gewähren, da sie nicht von einheitlicher Software im Klassenzimmer ausgehen können. Gleichzeitig können sich ihnen vielfältige Möglichkeiten der Integration digitaler Medien eröffnen; in der vorliegenden Fallstudie beispielsweise schätzen Lehrpersonen die Vielfalt, da sich daraus kreative Szenarien ergeben. Digitale Medien und deren Einsatz haben damit auf den ersten Blick das Potenzial, Unterrichtsentwicklung anzuregen.

Darüber hinaus sind Lehrpersonen durch die Integration disperser digitaler Medien in den Unterricht mit veränderten Kompetenzanforderungen konfrontiert. Durch unterschiedliche Gerätetypen muss ein technisch-didaktisches Verständnis erworben werden, das über das Einzelmedium hinausgeht und eher die medieninhärenten Handlungsstrukturen beachtet. Somit sind Lehrpersonen immer wieder damit konfrontiert, mediendidaktische und medienpädagogische Handlungsoptionen im Unterricht auszubilden. Innerhalb der vorliegenden Fallstudie waren die Entwicklungsschritte nicht so einschneidend, da die Schule auf vorhandene Medienarbeit und damit auch Kompetenzen von Lehrpersonen durch ein vorangegangenes 1:1-Konzept aufbauen konnte. Ist dies nicht der Fall, so dass BYOD direkt ohne vorherige 1:1-Ausstattung in der Schule implementiert wird, wird vor allem in der Personalentwicklung eine grosse Herausforderung gesehen.

Implikationen kann der eigentlich unterrichtlich geprägte Ansatz von BYOD aber auch auf die schulische Organisationsentwicklung als Ganzes haben. Neben infrastrukturellen Massnahmen (beispielsweise WLAN im Gebäude oder Drucker im Klassenzimmer) sind auch Veränderungen im Bereich der Organisationskommunikation denkbar. Geplant eingesetzt, kann es durch die Integration privater Geräte zu einer Erweiterung der Kontakte Schule-Elternschaft und zu einer aktiven Beteiligung aller Schulakteure kommen: Eltern und Schülerinnen und Schüler übernehmen einen Teil der Verantwortung für die Geräte. BYOD als Konzept wirkt in der Schule, indem es Akteure der Schule miteinander in Gespräch bringt und es eine gemeinsame, von allen Beteiligten getragene Struktur benötigt, um wirksam zu werden - zu achten ist allerdings darauf, dass sich diese Gespräche nicht nur auf technische Aspekte beschränken, sondern vor allem die Verbesserung von Unterricht im Fokus steht. Gleichzeitig ist zu bedenken, dass Schulentwicklung nur bei der Offenheit für Veränderung auf allen Seiten gelingt. 


\section{Literatur}

BMBF - Bundesministerium für Bildung und Forschung (BMBF) Referat Digitale Medien und Informationsinfrastruktur, Hrsg. 2010. Kompetenzen in einer digital geprägten Kultur - Medienbildung für die Persönlichkeitsentwicklung, für gesellschaftliche Teilhabe und für die Entwicklung von Ausbildungs- und Erwerbsfähigkeit. http://www.bmbf.de/pub/kompetenzen_in_digitaler_kultur.pdf

Deutscher Bundestag. 2011. "Zweiter Zwischenbericht der Enquete-Kommission: 〈Internet und digitale Gesellschaft〉.» Medienkompetenz (Vol. 2010): http://www.bundestag.de/internetenquete/dokumentation/Medienkompetenz/ Zwischenbericht_Medienkompetenz_1707286.pdf

Eisenmann, Maria, Thomas Grimm. 2011. Heterogene Klassen - Differenzierung in Schule und Unterricht. Baltmannsweiler: Schneider Hohengehren.

Fatke, Reinhard. 2003. «Fallstudien in der Erziehungswissenschaft.» In Handbuch qualitative Forschungsmethoden in der Erziehungswissenschaft, hrsg. v. Barbara Friebertshäuser u. Annedore Prengel, S. 56-68. Weinheim: Juventa.

Fullan, Michael. 2001. Leading in a Culture of Change. San Francisco: Jossey-Bass. Heinen, Richard und Manfred Kresse. 2011. «Nutzung privater Hardware im Unterricht - Schülerinnen- und Schülerbefragung an einem Gymnasium.» In DeLFI 2011: Die 9. e-Learning Fachtagung Informatik, hrsg. v. Steffen Friedrich, Andrea Kienle u. Holger Rohland, S. 31-39. Dresden: TUDpress.

Häuptle, Eva. 2006. Notebook-Klassen an einer Hauptschule: Eine Einzelfallstudie zur Wirkung eines Notebook-Einsatzes auf Unterricht, Schülerinnen und Schüler und Schule. Augsburg: Universität Augsburg. http://www.opus-bayern.de/ uni-augsburg/volltexte/2007/594/

Häuptle, Eva und Gabi Reinmann. 2006. Notebooks in der Hauptschule: Eine Einzelfallstudie zur Wirkung des Notebook-Einsatzes auf Unterricht, Lernen und Schule. Im Auftrag der Stiftung Bildungspakt Bayern, Abschlussbericht - kompakt: http://medienpaedagogik.phil.uni-augsburg.de/downloads/dokumente/2006/Notebook-Klassen_Abschlussbericht.pdf. Korrigiert: http://www. bildungspakt-bayern.de/uploads/media/Abschlussbericht.pdf

Kelle, Uwe, Susann Kluge. 2010. Vom Einzelfall zum Typus: Fallvergleich und Fallkontrastierung in der qualitativen Sozialforschung. Wiesbaden: VS Verlag.

Kerres, Michael, Richard Heinen und Jörg Stratmann. 2012. "Schulische IT-Infrastrukturen: Aktuelle Trends und ihre Implikationen für Schulentwicklung.» In Jahrbuch Medienpädagogik 9, hrsg. v. Renate Schulz-Zander, Birgit Eickelmann, Heinz Moser, Horst Niesyto u. Petra Grell, S. 161-174. Wiesbaden: VS Verlag.

Lampe, Andrea, Jeannette Rester, Wilfried Rüsse, Werner Sesink und Claudia Zentgraf. 2010. «PROJEKT «Schule Interaktiv - Transferı.» Abschlussbericht. Technische Universität Darmstadt. 
Ludwig, Luise, Kerstin Mayrberger und Adrian Weidmann. 2011. «Einsatz personalisierter iPads im Unterricht aus Perspektive der Schülerinnen und Schüler.» In DeLFI 2011: Die 9. e-Learning Fachtagung Informatik, hrsg. v. Steffen Friedrich, Andrea Kienle u. Holger Rohland, S. 7-17. Dresden: TUDPress.

MPFS - Medienpädagogischer Forschungsverbund Südwest. 2010. JIM-Studie 2010. Stuttgart. http://www.mpfs.de/fileadmin/JIM-pdf10/JIM2010.pdf

MPFS - Medienpädagogischer Forschungsverbund Südwest. 2011. JIM-Studie 2011. Stuttgart. http://www.mpfs.de/fileadmin/JIM-pdf11/JIM2011.pdf

Muno, Wolfgang. 2009. «Fallstudien und die vergleichende Methode.»In Methoden der vergleichenden Politik- und Sozialwissenschaft, hrsg. v. Susanne Pickel, Gert Pickel, Hans-Joachim Lauth u. Detlef Jahn, S. 13-131. Wiesbaden: VS Verlag für Sozialwissenschaften.

Nugroho, Dita und Michele Lonsdal. 2010. «Evaluation of OLPC programs globally: a literature review.» Australian Council for Educational Research: http:// wiki.laptop.org/images/a/a5/OLPC_Lit_Review_v4_Aug2010.pdf

Petko, Dominik. 2012. «Teachers' pedagogical beliefs and their use of digital media in classrooms: Sharpening the focus of the (will, skill, tool model and integrating teachers' constructivist orientations.» Computers \& Education 58 (4): 1351-1359.

Pierce, Jon L., Tatiana Kostova, Kurt T. Dirks. 2001. «Towards a theory of psychological ownership in organizations.» Academy of Management Review 26: 298-310.

Posch, Peter und Herbert Altrichter. 2009. «Schulen 2020 - Projektionen aufgrund gesellschaftlicher Entwicklungstendenzen.» In Schule 2020 aus Expertensicht: Zur Zukunft von Schule, Unterricht und Lehrerbildung, hrsg. v. Dorit Bosse u. Peter Posch, S. 31-37. Wiesbaden: VS Verlag für Sozialwissenschaft.

Prasse, Doreen und Wolfgang Scholl. 2001. «Wie funktioniert die Interneteinführung an Schulen? Die Rolle der Beteiligten und deren Zusammenarbeit: Idealund Problemtypen.» In Das Internet und die Schule: bisherige Erfahrungen und Perspektiven für die Zukunft, hrsg. v. Rudolf Grober u. Miriam Dubi, S. 63-83. Bern: Verlag Huber.

Rogers, Everett M. 2005. Diffusions of Innovations. New York: Free Press.

Rolff, Hans-Günter. 2007. Studien zu einer Theorie der Schulentwicklung. Weinheim u. Basel: Beltz Verlag

Schaumburg, Heike. 2002. Konstruktivistischer Unterricht mit Laptops? Eine Fallstudie zum Einfluss mobiler Computer auf die Methodik des Unterrichts. Dissertation. Berlin: Freie Universität Berlin. Zugriff am 30.11.2007 unter URL: http://www.diss.fu-berlin. de/2003/63/

Sandholtz, Judith H., Cathy Ringstaff, Davic C. Dwyer. 1997. Teaching with Technology: Creating Student Centred Classrooms. New York: Teachers College Press. 
Schnoor, Detlev. 1998. "Schulentwicklung durch Neue Medien: Lernort Multimedia.» In Jahrbuch Telekommunikation und Gesellschaft 1998, hrsg. v. Herbert Kubicek, Hans-Joachim Braczyk, Dieter Klumpp, Günter Müller u. a., S. 99-108. Heidelberg: R. V. Decker.

Schulz-Zander, Renate. 2001. "Neue Medien als Bestandteil von Schulentwicklung.» In Jahrbuch der Medienpädagogik, hrsg. v. Stefan Aufenanger, Renate Schulz-Zander u. Dieter Spanhel, S. 263-281. Opladen: Leske + Budrich.

Vorndran, Oliver und Detlev Schnoor. 2003. Schulen für Wissensgesellschaft: Ergebnisse des Netzwerkes Medienschulen. Gütersloh: Verlag Bertelsmann Stiftung. http://www.bertelsmann-stiftung.de/bst/de/media/xcms_bst_dms_ 15221_15222_2.pdf

Weinreich, Frank und Renate Schulz-Zander. 2000. «Schulen ans Netz - Ergebnisse der bundesweiten Evaluation. Ergebnisse einer Befragung der Computerkoordinatoren und -koordinatorinnen an Schulen.» Zeitschrift für Erziehungswissenschaften 4: 577-593.

\section{Anhang}

Folgende Codes wurden im Rahmen dieser Analyse entwickelt:

- Medienkompetenzerwerb

- Medienkompetenz Lehrer/innen

- Medienkompetenz Schüler und Schülerinnen

- Medienumgang / Nutzung im Unterricht

- Medienumgang / Nutzung im Unterricht / Einsatz anderer Medien

- Medienumgang / Nutzung im Unterricht / Einsatz anderer Medien / Handyverbot

- Medienumgang / Nutzung im Unterricht / Verwendung / Einsatz

- Medienumgang / Nutzung im Unterricht / Verwendung / Einsatz / Häufigkeit der Nutzung

- Medienumgang in der Freizeit

- Medienumgang in der Freizeit / soziale Netzwerke

- Probleme

- Probleme / Lösungsstrategien

- Projektbeurteilung / Feedback

- Projektbeurteilung / Feedback / Schülerinnen und Schülereinschätzung

- Relevanz digitaler Medien

- Rollen / Rollenveränderung

- Rollen / Rollenveränderung / Kompetenzveränderung / Lehrer

- soziale Spannungen

- Technische Ausstattung der Schule

- technisches Verständnis / Lehrer/innen 
- Unsicherheit / Verunsicherung durch Heterogenität der Geräte

- Unterrichtsplanung

- Unterschied Schullaptop / Privatlaptop

- Unterschied Schullaptop / Privatlaptop / Laptopnutzung

- Unterschied Schullaptop / Privatlaptop / Nachteile

- Unterschied Schullaptop / Privatlaptop / Vorteile

- Verhältnis und Zusammenarbeit Lehrer / Schülerinnen und Schüler

- Verhältnis und Zusammenarbeit Lehrer / Schülerinnen und Schüler / Verbote 